\title{
Two-dimensional non-Hermitian delocalization transition as a probe for the localization length
}

\author{
Tsunenao Kuwae ${ }^{1}$ and Nobuhiko Taniguchi ${ }^{2}$ \\ ${ }^{1}$ Department of Physical Electronics, Hiroshima University, Kagamiyama, Higashi-Hiroshima 739-8527, Japan \\ ${ }^{2}$ Institute of Physics, University of Tsukuba, Tennodai, Tsukuba 305-8571, Japan
}

(Received 14 September 2001; published 5 November 2001)

\begin{abstract}
When one applies a type of non-Hermitian effect, constant imaginary vector potential, to disordered systems, delocalization is induced even in two or lower dimensions. By using the non-Hermitian induced transition as a probe, we propose a procedure of estimating localization in arbitrary-dimensional systems. By examining numerically the two-dimensional non-Hermitian tight-binding model with onsite disorder, it is shown that the failure of absorbing the non-Hermitian effect, namely the breakdown of the imaginary gauge transformation, characterize the inverse localization length near the band center.
\end{abstract}

DOI: 10.1103/PhysRevB.64.201321

PACS number(s): 72.15.Rn, 73.20.Fz, 73.20.Jc

In noninteracting disordered systems with time-reversal symmetry, all the states are believed to be localized in two or lower dimensions. Often one encounters situations where non-Hermitian effect emerges effectively through coupling with the reservoir, interaction between particles, or nonequilibrium relaxation processes. This type of non-Hermitian effect is taken into account as a finite inelastic scattering time, hence following the scaling theory idea, localization will be truncated halfway only to exhibit a gradual crossover into metallic behavior. Recently, in a vortex pinning problem in a bulk superconductor by columnar defects, it has been stressed that the non-Hermitian term coupled with the firstorder differential, i.e., the imaginary vector potential, gives a fundamentally different effect on localization states in disordered systems. ${ }^{1}$ The term appears to take account for the in-plane magnetic field component. Along with subsequent numerical and analytical studies, ${ }^{2-4}$ it has been recognized that sufficiently large constant imaginary vector potential induces states with complex eigenenergies even in one dimension. The phenomenon has later been understood as a nonHermitian induced delocalization transition not a crossover. The same class of non-Hermitian terms is ubiquitous in diverse problems: classical (elastic, electromagnetic, or acoustic) wave propagation with absorptive medium, ${ }^{5}$ random walks in random media with drifting forces, ${ }^{6}$ or Burgers turbulence through a Cole-Hopf transformation. ${ }^{7}$

While non-Hermitian effect is usually hard to control, the strength of the imaginary vector potential is relatively easy to regulate not only in numerical studies but also in experiments. For instance, one changes the tilt angle of the magnetic field in the vortex pinning problem, or tunes an incident frequency and change the absorption rate in electromagnetic wave propagation. It has been suggested that the nonHermitian induced delocalization process can be detected by the energy spectrum. So with its drastic consequence on localization, the phenomenon provides a great opportunity to investigate the localization property without resorting to any transport phenomenon, i.e., neither attaching leads nor coupling with the reservoir.

In this Rapid Communication, pursuing the above possibility, we propose a procedure [Eq. (2) below] appropriate to extract the inverse localization length out of the nonHermitian dependence of the energy spectrum. Whereas the connection between the average density of states (DOS) and the localization length has been successfully managed in one dimension, it is not the case in higher dimensions ${ }^{8}$ (see the argument below). Our proposed procedure is principally applicable in arbitrary-dimensional systems. By using the twodimensional (2D) non-Hermitian model with time-reversal symmetry, we will confirm our proposal numerically. The non-Hermitian delocalization in 2D systems is far from complete understanding, and it is still at issue whether the transition is continuous or abrupt. ${ }^{9,10}$ On this regard, we also briefly mention our point of view based on numerical results.

The Hamiltonian considered for numerical calculations is the $d$-dimensional non-Hermitian tight-binding model,

$$
H(\boldsymbol{h})=-\frac{t}{2} \sum_{(i, j)} \exp \left(\boldsymbol{h} \cdot \boldsymbol{r}_{i j}\right)|i\rangle\left\langle j\left|+\sum_{i} V_{i}\right| i\right\rangle\langle i|,
$$

where $\boldsymbol{h}$ is the directed hopping, i.e., a lattice analog of the imaginary vector potential. $\left\{V_{i}\right\}$ is a random on-site potential chosen from a box distribution over $(-W, W) .|i\rangle$ denotes the state bound at $\boldsymbol{r}_{i}$, and the lattice summation $(i, j)$ is restricted over nearest-neighboring pairs of sites. We work on the two-dimensional case, where we particularly choose $\boldsymbol{h}$ as $h_{x}=h_{y}=h$ to avoid unnecessary troubles from lattice periodicity. In the rest of the paper, we adopt the convention of the unit lattice constant and $t=2$ as well as $\hbar=1$. Using an exact diagonalization technique, we have calculated a complete set of eigenvalues and eigenwavefunctions on a twodimensional $20 \times 20$ lattice. (Eigenfunctions are needed later to justify our interpretation.) Ensemble average is taken over 40 samples.

The possibility to detect localization by using the nonHermitian dependence of the spectrum has already been noticed in Ref. 1. The reasoning is as follows. Suppose an eigenstate $\phi_{\boldsymbol{h}=\mathbf{0}}(\boldsymbol{r})$ is localized exponentially around $\boldsymbol{r}_{0}$ with the localization length $\xi$, that is, $\phi_{0} \sim e^{-\left|\boldsymbol{r}-\boldsymbol{r}_{0}\right| / \xi}$. When one turns on $\boldsymbol{h}$ gradually, its eigenenergy does not change in value, while the right eigenwavefunction progresses following the imaginary gauge transformation $\phi_{\boldsymbol{h}}(\boldsymbol{r})$ $\sim e^{\boldsymbol{h} \cdot\left(\boldsymbol{r}-\boldsymbol{r}_{0}\right)} \phi_{\mathbf{0}}(\boldsymbol{r})$ up to a small correction by the boundary condition. Such "absorption" of the non-Hermitian influence 


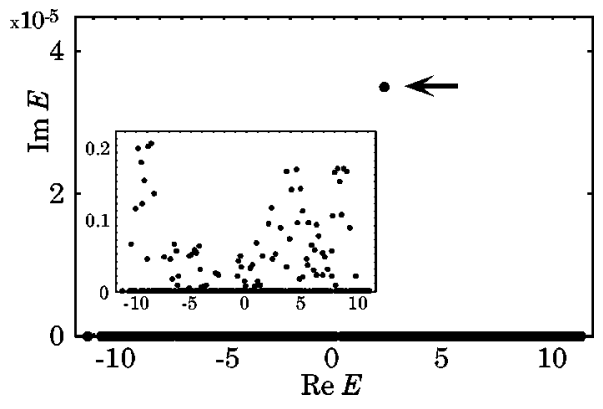

FIG. 1. A typical example of the energy spectrum for a given configuration of random site energies (only the upper half plane shown here). Random on-site potential is chosen from a box distribution $[-10,10]$. The value of $h$ is adjusted to $h=0.21$, where only one pair of states (indicated by the arrow) get complex. Inset: A typical structure of the DOS for a larger $h(h=0.60)$. Both localized and delocalized states are mixed up in the same energy range.

on the spectrum can take effect only when $\boldsymbol{h}$ is smaller than the inverse localization length. Beyond that, the state undergoes such a drastic change that it transforms into a complex energy (delocalized) state. Putting it the other way round, one uses the breakdown of the imaginary gauge transformation to characterize and give a practical estimate of the inverse localization length.

A naive way to estimate the breakdown of the imaginary gauge transformation is to examine the average DOS defined on the complex energy plane, $\rho_{h}(E, \mathcal{E})=\left\langle\delta\left(E-\operatorname{Re} E_{n}\right) \delta(\mathcal{E}\right.$ $\left.\left.-\operatorname{Im} E_{n}\right)\right\rangle$. When all the states are localized at sufficiently small $h$, energies are all real and $\rho_{h}(E, \mathcal{E}) \propto \delta(\mathcal{E})$. With increasing $h$, the support of $\rho_{h}(E, \mathcal{E})$ extends into a $\mathcal{E} \neq 0$ region in the presence of complex energy states. By defining $h_{0}(E)$ by the point having a finite density at $\mathcal{E} \neq 0$ for each $E$ one sees $h_{0}(E)$ indicates the breakdown of the imaginary gauge transformation. Accordingly, it is suggested that the inverse localization length may be estimated by the formula $1 / \xi(E)=h_{0}(E)$. $^{1,12}$

While the above argument is conceptually simple and attractive, the estimate of the localization length by using the average DOS has been successful only in one dimension, whose success is traced to a special structure of the energy spectrum. In one dimension, irrespective of the value of $h$, the support of $\rho_{h}(E, \mathcal{E})$ becomes one-dimensional on the complex energy plane, i.e., consisting of two line segments along the real axis plus a "bubble" part in-between corresponding to complex energy states. ${ }^{1-4}$ The shape of the bubble turns out quite insensitive to disorder configurations. Because of it, one can readily read off the value of $h_{0}(E)$ from the endpoints of the arcs of the bubble.

By contrast, the DOS support on higher dimensions is no longer one-dimensional but much more involved. A typical structure of the two-dimensional DOS for a sufficiently large $h$ is shown in the inset of Fig. 1. As one can see, localized and delocalized states coexist unpredictably over the entire energy range, showing that DOS suffers large fluctuations. The system has no clear-cut mobility edge distinguishing between real-energy (localized) and complex-energy (delocalized) states. In fact, judging from existing numerical ${ }^{1}$ and analytical $^{13}$ results, it is very likely that the average DOS



FIG. 2. An evolution of the first pair of levels turning complex values, by increasing $h$. In this configuration, we assign the critical value $h_{c}$ as $h_{c}=0.21$. The $\operatorname{Im} E=0$ plane is drawn as a shaded plane for a guide to the eye.

$\rho(E, \mathcal{E})$ always retains a finite contribution proportional to $\delta(\mathcal{E})$ hence real energy states persist in existing for sufficiently large $h$, even near the band center. The feature of $\rho_{h}(E, \mathcal{E})$ in higher dimensions makes it highly dubious to seek the breakdown of the imaginary gauge transformation at a fixed $E$. Moreover, numerical calculations on twodimensional systems suggest that $h_{0}(E)$ will estimate an inverse localization length at more than one order of magnitude larger than that obtained by any other method such as the recursion method. ${ }^{14}$

Resolving problems above to answer whether the nonHermitian effect characterizes the localization length or not, we propose an alternative criterion to extract the localization length. Our solution is to find the breakdown of the imaginary gauge transformation in a more faithful and careful way: First we identify the smallest directed hopping strength $h_{c}$ where any of the eigenenergies gets complex in a certain configuration of the random potential (see Fig. 1), and next we take the ensemble average of $h_{c}$ to obtain the inverse localization length by ${ }^{11}$

$$
\left\langle h_{c}\right\rangle=1 / \xi(0)
$$

The estimated localization length corresponds to that around the band center, since states near the band center tend to be delocalized first. Equation (2) and the procedure leading to it is our main result in the paper. The advantage of Eq. (2) is clear. In any localized system, we can assign an unambiguous value of $h_{c}$ for each configuration, and perform the ensemble average of $h_{c}$. It should be stressed that although there is no discrepancy between $\left\langle h_{c}\right\rangle$ and $h_{0}(0)$ for one dimension, $\left\langle h_{c}\right\rangle$ becomes much smaller than $h_{0}(0)$ in higher dimensions, since the latter cannot detect a weakly-broken situation as in Fig. 1. Unlike $h_{0}(E)$ (if at all correctly defined in higher dimensions), we cannot obtain $\left\langle h_{c}\right\rangle$ from the average DOS.

It is worth discussing a little more the definition of $h_{c}$ and finite volume effect. Although we have initially intended to assign $h_{c}$ as the point where the imaginary gauge transformation fails to absorb all the non-Hermitian effect, we should keep in mind that such a mechanism works only approximately in a finite system. Figure 2 represents a typical evolution of the first pair of levels getting complex. It shows 


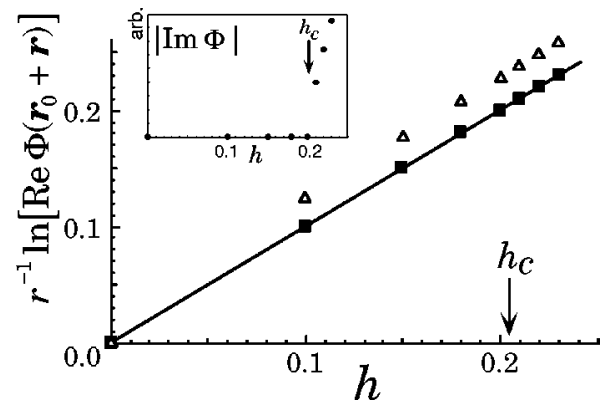

FIG. 3. The validity of the imaginary gauge transformation in the right wave function. $r^{-1} \log \left|\operatorname{Re} \Phi\left(\boldsymbol{r}_{0}+\boldsymbol{r}\right)\right|$ is plotted as a function of the imaginary vector potential $h$ where $\boldsymbol{r}_{0}$ is the localization center. The value is normalized by that at $h=0$. Squares refer to the values at $r=1$, and open triangles to those at $r=9$. A solid line is what is expected by the imaginary gauge transformation. Inset: $\left|\operatorname{Im} \Phi\left(\boldsymbol{r}_{0}+\boldsymbol{r}\right)\right|$ for $r=1$ as a function of $h$. A jump at $h_{c}$ is clearly observed.

that complex energy states appear through merging two adjacent levels, but well before it, the pair of levels shift gradually without gaining any imaginary part. Strictly speaking, the effect of $h$ partially remains for any small $h$, hence the gauge transformation is already broken down for infinitesimal $h$. Nevertheless, we assign the value of $h_{c}$ by a merging point. The shift of energies before $h_{c}$ arises from finite-size effect, the consistency with the periodic boundary condition in a finite system.

To justify the statement above, Fig. 3 illustrates the spatial dependence of the right eigenwavefunction $\phi(\boldsymbol{r})$ of the corresponding levels of Fig. 1. When we look at the amplitude next to a localization center, it evolves precisely in the form predicted by the imaginary gauge transformation (the solid line in the figure). However, such dependence fails appreciably at a point sufficiently far away from a localization center ( $r=9$ on a $20 \times 20$ lattice). The deviation is responsible for the level shift observed in $h<h_{c}$, and has nothing to do with any precursor to delocalization. Simultaneously, Fig. 3 reveals clearly that it is almost impossible, at least numerically, to detect the point $h_{c}$ by examining the spatial dependence of the wave function amplitude, in contrast to the onedimensional system. ${ }^{15}$ The overall shape is barely changed through the critical value $h_{c}$ since the contribution of the imaginary part is too small to give a visible deviation (see the inset). It is remarked that on a few occasions some abrupt movement of the localized center is observed before reaching $h_{c}$ without any finite imaginary part. We still find, however, the spatial dependence following the gauge transformation close to a localization center both before and after it, so the phenomenon should be attributed as well to an artifact of finite-system size. ${ }^{16}$

To obtain $h_{c}$ from numerical data, we need to distinguish between energies with vanishing and nonvanishing imaginary parts. Such type of estimate is usually hard in numerics, but in our problem, we can unambiguously identify it, because complex energy states always emerge by pairs (see Fig. 2).

Following the procedure leading to Eq. (2), we find $h_{c}$ for a given configuration of the random potential, and take the

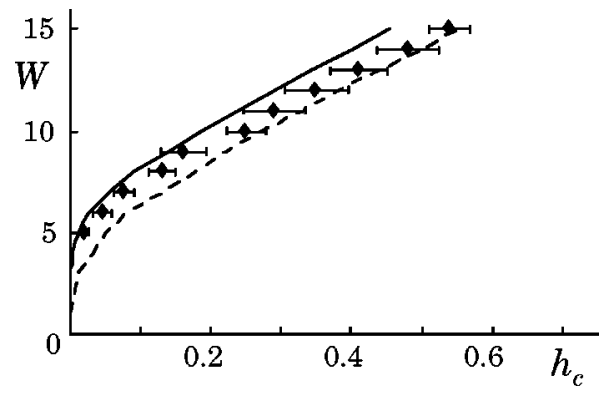

FIG. 4. $\left\langle h_{c}\right\rangle$ versus the disorder strength $W$ to confirm Eq. (2). Data points $(\diamond)$ are obtained from 40 ensembles of a $20 \times 20$ lattice. The solid line is the inverse localization length for a $\infty \times \infty$ lattice, and the dashed line for a $16 \times \infty$ one. Excerpted from Ref. 14.

ensemble average to obtain the inverse localization length. Our numerical results are compared with the ones obtained from the recursion method combined with finite scaling analysis, ${ }^{14}$ which is known among the most reliable estimates of the localization length. Since our numerical parameters reside in a strongly localized region, we take the Lyapunov exponent directly as the inverse localization length with little finite size correction.

Figure 4 shows the ensemble-averaged value $\left\langle h_{c}\right\rangle$ and its variance from a $20 \times 20$ lattice, compared with the inverse localization length for a $\infty \times \infty$ lattice denoted by the solid line (for $16 \times \infty$ lattice by the dashed line) excerpted from Ref. 14. The agreement between $\left\langle h_{c}\right\rangle$ and the inverse localization is clear, and the variance $\delta h_{c}$ is relatively small, considering the fact we work on a comparatively small system size and ensembles. This shows the effectiveness of the present approach. Our data points show a systematic drift, though relatively small, from the $\infty \times \infty$ line to the $16 \times \infty$ line in approaching $h_{c} \rightarrow 1$. The tendency is consistent because the finite-size effect becomes increasingly prominent in this limit. It is emphasized that the agreement is substantial and the ability to predict the localization length quantitatively is the virtue of Eq. (2), while other numerical objects such as the participation ratio are often lacking.

As for the nature of the two-dimensional non-Hermitian delocalization transition, our view based on the present numerical results is as follows. By attributing the level shift in the region $h<h_{c}$ to finite-size effect, it is natural to infer that the transition is not continuous but abrupt in the limit of infinite size at least for a given configuration. By assuming further the self-averaging property, one is tempted to reach the conclusion that the two-dimensional non-Hermitian delocalization transition is abrupt for bulk systems. It should be recalled, however, that in our particular choice of averaging procedure, we hardly respect the additional symmetry present at the band center. So we believe as a possible scenario, that the transition away from the band center is discontinuous but continuous at the band center, which is consistent both with the nonperturbative analysis by the supermatrix ${ }^{10}$ and with the symmetry argument applicable to the band center. ${ }^{9}$ However, the present results are not sufficient to conclude this clearly, and it requires further studies in the future to draw a decisive conclusion on the issue. 
In conclusion, we have shown, to the best of our knowledge, for the first time how the two-dimensional localization can be probed by using the non-Hermitian transition induced by the imaginary vector potential, proposing a practical procedure to make an estimation of the inverse localization length. By checking the level shifting through the transition, it has been understood clearly that the two-dimensional nonHermitian delocalization transition occurs through merging adjacent levels. It is true at present that the evaluation of the localization length by using the non-Hermitian effect has not yet fulfilled such accuracy as the recursion method enjoys, and it needs much more labor or fine tuning to find $h_{c}$. However, it surely serves as a useful alternative that one may be able to apply even in experimental settings. Furthermore, as the present method need not increase the system size, it may be advantageous in numerical studies on more complicated systems such as the disordered interacting systems.

We acknowledge the support in the initial stage of the research from the Grant-in-Aid for Scientific research No. 11216204 by Japanese Ministry of Education.
${ }^{1}$ N. Hatano and D.R. Nelson, Phys. Rev. Lett. 77, 570 (1996); Phys. Rev. B 56, 8651 (1997).

${ }^{2}$ P.W. Brouwer, P.G. Silvestrov, and C.W.J. Beenakker, Phys. Rev. B 56, R4333 (1997).

${ }^{3}$ I.Y. Goldsheid and B.A. Khruzhenko, Phys. Rev. Lett. 80, 2897 (1998).

${ }^{4}$ P.G. Silvestrov, Phys. Rev. B 58, R10 111 (1998).

${ }^{5}$ V. Freilikher, M. Pustilik, and I. Yurkevich, Phys. Rev. Lett. 73, 810 (1994); Phys. Rev. B 50, 6017 (1994).

${ }^{6}$ D. Fisher et al., Phys. Rev. A 31, 3841 (1985); V.E. Kravtsov, I.V. Lerner, and V.I. Yudson, Zh. Éksp. Teor. Fiz. 91, 569 (1986) [Sov. Phys. JETP 64, 336 (1986)].

${ }^{7}$ J.P. Bouchaud, M. Mezard, and G. Parisi, Phys. Rev. E 52, 3656 (1995).
${ }^{8}$ C. Mudry et al., Phys. Rev. B 58, 13539 (1998).

${ }^{9}$ C. Mudry, B.D. Simons, and A. Altland, Phys. Rev. Lett. 80, 4257 (1998).

${ }^{10}$ A.V. Kolesnikov and K.B. Efetov, Phys. Rev. Lett. 84, 5600 (2000).

${ }^{11} \mathrm{By}$ convention of numerical studies, the localization length is referred to that along the lattice direction. So the choice of $\boldsymbol{h}$ $=h(1,1)$ makes the $\sqrt{2}$ factor disappear in the following Eq. (2).

${ }^{12}$ V. Gurarie and A. Zee, cond-mat/9802042 (unpublished); E. Brézin and A. Zee, Nucl. Phys. B 509, 599 (1998).

${ }^{13}$ K.B. Efetov, Phys. Rev. Lett. 79, 491 (1997).

${ }^{14}$ A. MacKinnon and B. Kramer, Z. Phys. B 53, 1 (1983).

${ }^{15}$ N. Hatano and D.R. Nelson, Phys. Rev. B 58, 8384 (1998).

${ }^{16} \mathrm{~T}$. Kuwae and N. Taniguchi (private communication). 